University of Montana

ScholarWorks at University of Montana

Ecosystem and Conservation Sciences Faculty

Publications

Ecosystem and Conservation Sciences

2011

\title{
An Ecosystem-Scale Model for the Spread of a Host-Specific Forest Pathogen in the Greater Yellowstone Ecosystem
}

Jaclyn A. Hatala

Michael C. Dietze

Robert L. Crabtree

Katherine Kendall

Diana Six

University of Montana - Missoula, diana.six@umontana.edu

See next page for additional authors

Follow this and additional works at: https://scholarworks.umt.edu/decs_pubs

Part of the Ecology and Evolutionary Biology Commons

Let us know how access to this document benefits you.

\section{Recommended Citation}

Hatala, Jaclyn A.; Dietze, Michael C.; Crabtree, Robert L.; Kendall, Katherine; Six, Diana; and Moorcroft, Paul R., "An Ecosystem-Scale Model for the Spread of a Host-Specific Forest Pathogen in the Greater Yellowstone Ecosystem" (2011). Ecosystem and Conservation Sciences Faculty Publications. 40. https://scholarworks.umt.edu/decs_pubs/40

This Article is brought to you for free and open access by the Ecosystem and Conservation Sciences at ScholarWorks at University of Montana. It has been accepted for inclusion in Ecosystem and Conservation Sciences Faculty Publications by an authorized administrator of ScholarWorks at University of Montana. For more information, please contact scholarworks@mso.umt.edu. 


\section{Authors}

Jaclyn A. Hatala, Michael C. Dietze, Robert L. Crabtree, Katherine Kendall, Diana Six, and Paul R. Moorcroft 


\title{
An ecosystem-scale model for the spread of a host-specific forest pathogen in the Greater Yellowstone Ecosystem
}

\author{
Jaclyn A. Hatala, ${ }^{1,7}$ Michael C. Dietze, ${ }^{2}$ Robert L. Crabtree, ${ }^{3}$ Katherine Kendall,${ }^{4}$ Diana Six, ${ }^{5}$ \\ And Paul R. MoOrcroft ${ }^{6}$ \\ ${ }^{1}$ Department of Environmental Science, Policy, and Management, University of California, Berkeley, California 94720 USA \\ ${ }^{2}$ Department of Plant Science, University of Illinois, Urbana, Illinois 61801 USA \\ ${ }^{3}$ Yellowstone Ecological Research Center, Bozeman, Montana 59718 USA \\ ${ }^{4}$ Northern Rocky Mountain Science Center, U.S. Geological Survey, West Glacier, Montana 59936 USA \\ ${ }^{5}$ College of Forestry and Conservation, University of Montana, Missoula, Montana 59812 USA \\ ${ }^{6}$ Department of Organismic and Evolutionary Biology, Harvard University, Cambridge, Massachusetts 02138 USA
}

Abstract. The introduction of nonnative pathogens is altering the scale, magnitude, and persistence of forest disturbance regimes in the western United States. In the high-altitude whitebark pine (Pinus albicaulis) forests of the Greater Yellowstone Ecosystem (GYE), white pine blister rust (Cronartium ribicola) is an introduced fungal pathogen that is now the principal cause of tree mortality in many locations. Although blister rust eradication has failed in the past, there is nonetheless substantial interest in monitoring the disease and its rate of progression in order to predict the future impact of forest disturbances within this critical ecosystem.

This study integrates data from five different field-monitoring campaigns from 1968 to 2008 to create a blister rust infection model for sites located throughout the GYE. Our model parameterizes the past rates of blister rust spread in order to project its future impact on highaltitude whitebark pine forests. Because the process of blister rust infection and mortality of individuals occurs over the time frame of many years, the model in this paper operates on a yearly time step and defines a series of whitebark pine infection classes: susceptible, slightly infected, moderately infected, and dead. In our analysis, we evaluate four different infection models that compare local vs. global density dependence on the dynamics of blister rust infection. We compare models in which blister rust infection is: (1) independent of the density of infected trees, (2) locally density-dependent, (3) locally density-dependent with a static global infection rate among all sites, and (4) both locally and globally density-dependent. Model evaluation through the predictive loss criterion for Bayesian analysis supports the model that is both locally and globally density-dependent. Using this best-fit model, we predicted the average residence times for the four stages of blister rust infection in our model, and we found that, on average, whitebark pine trees within the GYE remain susceptible for 6.7 years, take 10.9 years to transition from slightly infected to moderately infected, and take 9.4 years to transition from moderately infected to dead. Using our best-fit model, we project the future levels of blister rust infestation in the GYE at critical sites over the next 20 years.

Key words: alternate hosts; Bayesian statistics; blister rust; Cronartium ribicola; Greater Yellowstone Ecosystem; nonnative pathogen; pathogen modeling; Pinus albicaulis; whitebark pine.

\section{INTRODUCTION}

The role of humans in altering ecosystem disturbances has increased dramatically over the last century, especially in the western United States, where introduced pathogens are creating novel disturbance regimes (Logan et al. 2003, Ellison et al. 2005). As a result, the economic and ecological consequences of the introduction of nonnative forest pathogens have presented a tremendous challenge for forest managers (Brown and Hovmoller 2002). The introduction of white pine blister

Manuscript received 13 November 2009; revised 8 June 2010; accepted 9 July 2010; final version received 8 September 2010. Corresponding Editor: A. D. McGuire.

${ }^{7}$ E-mail: jhatala@berkeley.edu rust (Cronartium ribicola), which is a now a principal cause of mortality within whitebark pine (Pinus albicaulis) populations at high altitudes in the Greater Yellowstone Ecosystem (GYE), serves as a dramatic and devastating example of the effects of an introduced pathogen on North American forests (Kendall and Arno 1990).

White pine blister rust is a nonnative fungal pathogen that was introduced to North America near British Columbia in 1910 on a stock of white pine imported for plantation (Spaulding 1922, Maloy 1997). It propagates through two obligate alternate hosts: five-needled pines and either shrubs belonging to the genus Ribes or select herbaceous species within the family Orobanchaceae: scarlet Indian paintbrush (Castilleja miniata), sickletop 
lousewort (Pedicularis racemosa), and bracted lousewort (Pedicularis bracteosa). Despite a continental-scale federal program to eradicate white pine blister rust from the U.S. landscape that began in the 1930s and ran until 1965, white pine blister rust still pervades highaltitude five-needled pine forests within much of the western United States (Smith and Hoffman 2000).

Pathogens such as white pine blister rust operate through mechanisms that function at the cellular level, yet they produce disturbance patterns with cascading effects that scale up to measurable changes throughout the landscape (Castello et al. 1995, Moorcroft et al. 2006). Pathogen disturbances in forests can produce dramatic ecosystem-scale effects through the reorganization of community structure (Frelich and Reich 1999) in addition to the alteration of abiotic processes such as hydrology and soil composition (Ellison et al. 2005). Consequently, ecologists have identified many links between pathosystem dynamics, which describe the ecological connections between pathogen and host populations, and biotic heterogeneity, such as landscape connectivity patterns, as well as with abiotic heterogeneity, such as topography (Holdenrieder et al. 2004). Epidemiology models at the landscape scale have indicated that the spatial extent (O'Neill et al. 1992) and rate (Park et al. 2002) of pathogen-caused disturbance interact through feedbacks, with spatially explicit landscape patterns at both the local and regional scales (Park et al. 2001). Because whitebark pine is a keystone species within high-altitude ecosystems (Tomback et al. 2001), and because pathogens often operate in ways that are spatially explicit, we expected that spatial environmental covariates, driven by differences in elevation, slope, and aspect within these highaltitude environments, might predict the landscape-level population dynamics of the historic spread of white pine blister rust in whitebark pine within the GYE.

In this analysis, we constructed a metapopulation model for the spread of blister rust in whitebark pine, based on field surveys collected between 1968 and 2008. This analysis used a data set aggregated from five different monitoring campaigns to estimate transitions between four stages of white pine blister rust infection (susceptible, slight infection, moderate infection, and dead) at 121 sites throughout the GYE from 1968 to 2008. In the formulation of our model, we included a site-specific parameter that we used to compare differences between infection rates at different sites with possible environmental drivers of white pine blister rust dynamics. We created our infection model using a Bayesian statistical framework, which permitted the modeling of $95 \%$ confidence intervals for the four levels of infection in 1968-2008, where data exist, and also in our future projections of blister rust infection. Furthermore, the Bayesian framework of our model permitted the inclusion of informed priors, where we used the field experience of forest managers in the GYE to set an informed starting point for our parameters.

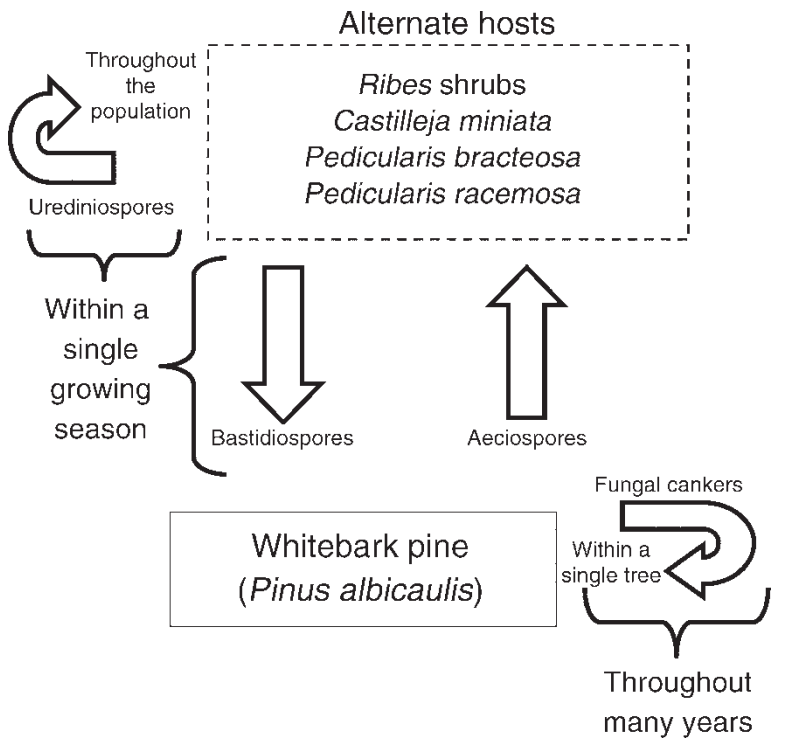

FIG. 1. Blister rust (Cronartium ribicola) spreads through two alternate hosts, members of the genus Ribes or select members of the family Orobanchaceae, and whitebark pine. The fungus can only overwinter in whitebark pine, so the spread from whitebark pine to an alternate host to another whitebark pine must occur within a single growing season. It is important to note that the fungus cannot spread from pine to pine, but must pass through an intermediate alternate host species.

Whitebark pine trees exist only at high elevations throughout the GYE, and patches of whitebark pine trees occur between distances where it is critical to analyze both local and global infection dynamics in a metapopulation structure. Within this work, we define local dynamics as infection that results from white pine blister rust within the same field site, whereas global dynamics are defined as infection that occurs between sites, regardless of distance. Using the infection model, we tested four hypotheses for the mechanistic population-level infection dynamics of blister rust operating within the GYE: (1) blister rust infection is independent of infected tree density, (2) blister rust is proportional to the local infected tree density, (3) blister rust infection is proportional to the local infected tree density, with a static background global infection rate, or (4) blister rust is proportional to both the local and global infected tree density. Although this model is parameterized with a data set based on current environmental conditions, it nonetheless yields key insights into the population dynamics of white pine blister rust infection, in addition to providing critical information regarding the residence time within each class of infection within the GYE based on the infection dynamics within the past 40 years.

\section{Methods \\ Pathology}

White pine blister rust has a complex life history, with five spore stages and two obligate alternate hosts (Arthur 1934), outlined in Fig. 1. White pine blister 


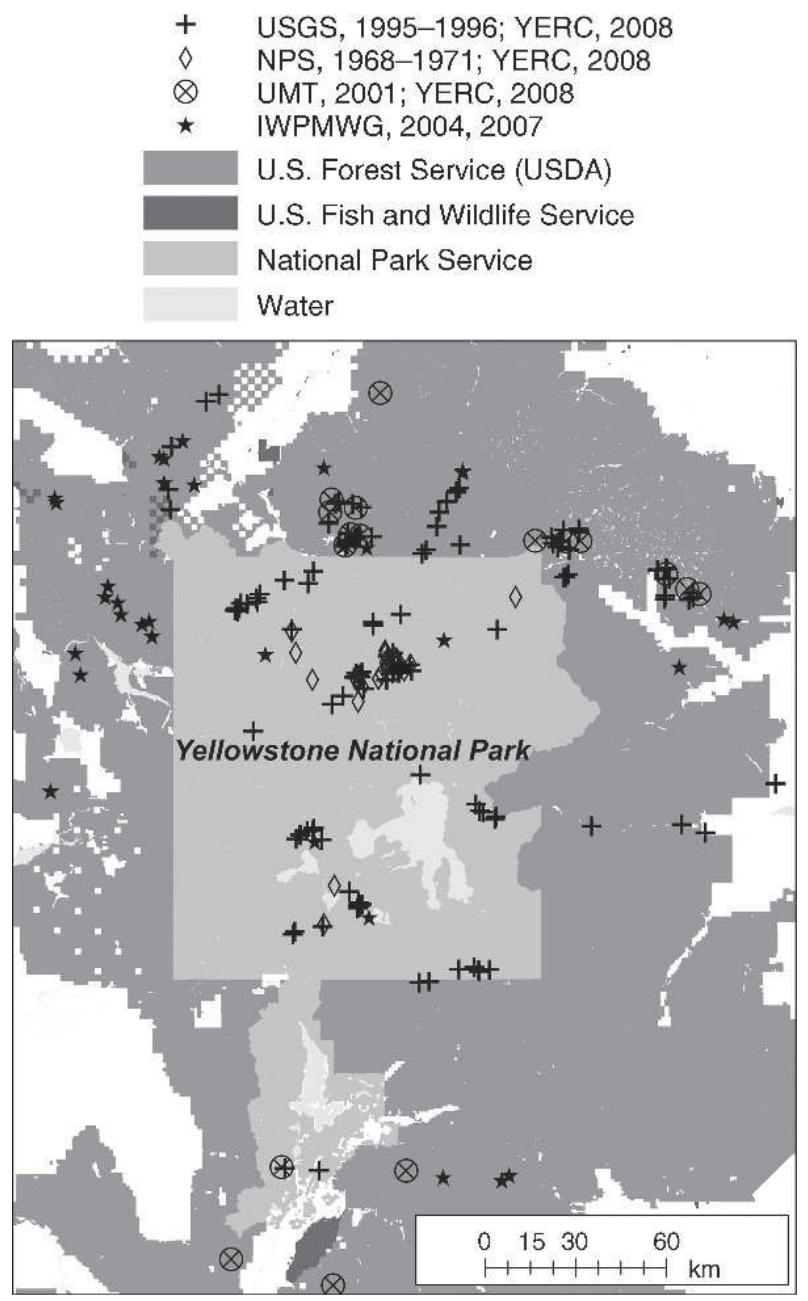

FIG. 2. For our study, blister rust field data are aggregated from five different sources across the Greater Yellowstone Ecosystem (GYE), USA, for the years 1968-2008. This data set contains much of the spatial variation within whitebark pine populations in the GYE. YERC is the Yellowstone Ecological Research Center in Bozeman, Montana. The data sets mapped here reflect those outlined in Table 1.

rust does not spread directly from one whitebark pine tree to another, but requires intermediate infection within an alternate host. The most numerous and widely distributed alternate hosts for blister rust within the GYE are various species of currants and gooseberries, which are members of the genus Ribes (Snell 1942). In Asia, which is the biogeographic origin of the blister rust pathosystem, plant species within the Orobanchaceae family have also been recognized as alternate hosts for blister rust (Yi and Kim 1983, McDonald et al. 2006), although field observations of white pine blister rust infection on species of Orobanchaceae have yet to be recorded within the GYE. In 2004, for the first time in North America, two additional alternate hosts in the Orobanchaceae plant family, the sickletop lousewort (Pedicularis racemosa) and scarlet Indian paintbrush (Castilleja miniata), were described as naturally occurring alternate hosts for blister rust in northern Idaho,
USA (McDonald et al. 2006). Blister rust alternate host infection was also described in bracted lousewort (Pedicularis bracteosa) in 2006 at the same field site in northern Idaho (Zambino et al. 2007). Whether these recent discoveries represent newly evolved strains of blister rust, new introductions of a different genotype, or simply that forest managers failed to previously recognize white pine blister rust infections on members of Orobanchaceae remains to be resolved.

Explicitly monitoring and modeling the intra-annual spread of white pine blister rust between its alternate hosts is difficult because there are $>17$ species of Ribes and at least three species of alternate hosts in the Orobanchaceae family that are prevalent within the GYE and would require intensive white pine blister rust surveys during a small temporal window of spore activity to determine the prevalence of infection. Therefore, within this study, we focus on the interannual progress of white pine blister rust infection, and our model calculates infection levels at an annual time step. By choosing an annual timescale, we do not explicitly model the infection within alternate host populations, because white pine blister rust can only overwinter within the woody tissue of whitebark pine. Although our model only represents blister rust infection within trees, we implicitly consider the impact of alternate hosts through our model formulations that consider locally density-dependent infection, described in the section Model formulation.

\section{Data}

For most forest pathogens, including white pine blister rust, it is difficult to observe the physical presence of the pathogen itself, and instead field data are collected on the symptoms exhibited by host individuals. The field data used in this analysis were gathered during surveys from 1968 to 2008 at irregular temporal intervals due to the large amount of time and resources required of highaltitude forest inventories. The field data are aggregated from five different sources: the Interagency Whitebark Pine Monitoring Group (IWPMG), the National Park Service (NPS), the U.S. Geological Survey (USGS) Northern Rocky Mountain Science Center, the University of Montana (UMT) College of Forestry and Conservation, and the Yellowstone Ecological Research Center (YERC). See Fig. 2 for the spatial distribution of study sites used in this analysis. The sampling protocols, summarized in Table 1, vary by study. In the formation of all study designs within the aggregate data set, sample plots or transects were randomly located within forest stands that were considered as "representative" for whitebark pine throughout the surrounding area. Thus, the combined data set overall could be biased toward sampling trees at the center of stands, and might be lacking data in areas with very low numbers of whitebark pine trees. Because many of the plots were located within the Yellowstone National Park boundary, plot locations could not be permanently marked and trees could not be 
TABLE 1. Five field protocols for data sets (1968-2008) assimilated into the metapopulation study of blister rust (Cronartium ribicola) infection in whitebark pine (Pinus albicaulis) forests of the Greater Yellowstone Ecosystem (GYE).

\begin{tabular}{|c|c|c|c|c|c|}
\hline $\begin{array}{l}\text { Data set } \\
\text { collector }\end{array}$ & Years & No. sites & Survey method & Site establishment method & Source \\
\hline IWPMG & 2004,2007 & 22 & $10 \times 50 \mathrm{~m}$ transects & $\begin{array}{l}\text { randomly located in the } \\
\text { GYE grizzly bear } \\
\text { Primary Conservation } \\
\text { Area, in whitebark pine } \\
\text { stands }>2.5 \text { ha }\end{array}$ & GYIWPMG (2007) \\
\hline NPS & $1968 / 1970,2008 \dagger$ & 19 & $\begin{array}{l}\text { 28-tree plots of } \\
\text { variable length } \\
\text { and width }\end{array}$ & $\begin{array}{l}\text { placed within Yellowstone } \\
\text { National Park in areas } \\
\text { of probable blister rust } \\
\text { infestation }\end{array}$ & $\begin{array}{l}\text { Yellowstone National } \\
\text { Park (1968-1971) }\end{array}$ \\
\hline USGS & $1995 / 1996,2008 \dagger$ & 136 & $\begin{array}{l}300 \text { foot long } \\
\text { variable-width } \\
\text { transects and } \\
0.1 \text {-acre plots }\end{array}$ & $\begin{array}{l}\text { dispersed throughout the } \\
\text { GYE to encompass the } \\
\text { widest variety of } \\
\text { whitebark pine habitat } \\
\text { possible }\end{array}$ & Kendall (1995) \\
\hline UMT & $2001 / 2002,2007 \dagger$ & 20 & $\begin{array}{l}\text { variable-length } \\
\text { transects } 10 \mathrm{~m} \\
\text { wide }\end{array}$ & $\begin{array}{l}\text { randomly located within } \\
\text { known whitebark pine } \\
\text { habitat }\end{array}$ & $\begin{array}{l}\text { Newcomb (2003), Six } \\
\text { and Newcomb (2005) }\end{array}$ \\
\hline YERC & 2000,2007 & 8 & $\begin{array}{l}10 \text { plots }(5.2 \mathrm{~m} \\
\text { radius) at each } \\
\text { site }\end{array}$ & $\begin{array}{l}\text { sites selected to fall within } \\
\text { hyperspectral imagery } \\
\text { flightline }\end{array}$ & Halligan et al. (2003) \\
\hline
\end{tabular}

Note: Collectors, top to bottom, are: Interagency Whitebark Pine Monitoring Working Group, Bozeman, Montana; National Park Service, Yellowstone National Park, Mammoth, Wyoming; U.S. Geological Survey, Northern Rocky Mountain Science Center, Bozeman, Montana; University of Montana, School of Forestry, Missoula, Montana; Yellowstone Ecological Research Center, Bozeman, Montana.

$\dagger$ Plots were re-censused in 2007/2008 by the Yellowstone Ecological Research Center, Bozeman, Montana, and the data are previously unpublished.

tagged, precluding the use of simpler demographic models that track the fate of individual trees. Accordingly, plot re-censuses were treated as repeated samples from a local population rather than re-measurement in the strict sense.

Tree evaluation protocols varied between study groups, but all protocols recorded the presence or absence of white pine blister rust symptoms, including bole cankers, fruiting bodies, and rodent chewing (indicating consumption of a canker) on individual trees. The NPS, USGS, and YERC protocols evaluated trees in four classes of white pine blister rust infection that were adopted as the four classes used for the infection model: susceptible, slightly infected, moderately infected, and dead (National Park Service 19681971). The UMT protocol recorded a more detailed 118 infection score for each tree (Six and Newcomb 2005). These 18 infection classes were translated into the four classes established in the YERC and USGS protocols by using a decision tree. The decision tree first separated uninfected trees into the susceptible class, and then separated the classes of infected trees by scores of less than 9 into the slightly infected class, scores of 915 into the moderately infected class, and scores of 1618 into the dead class. The IWPMG data set was developed using a more descriptive protocol that evaluated trees based on the number of cankers in each one-third of the tree branch area and in each one-third of the total tree bole. These data were also translated into four infection classes by a decision tree that first separated uninfected trees into the susceptible class and dead trees with signs of past blister rust infection into the dead class. The decision tree next separated trees with cankers in one-third or less of the total bole or branches into the slightly infected class, and trees with cankers in two-thirds or greater of the total bole or branches into the moderately infected class. Parsing the UMT and IWPMG data into these four classes by decision trees yielded a large, but simplified, data set that is ecologically informative for population-level study and computationally efficient. Bayesian modeling accommodates the differences in sampling methods between data sets, because observation error is modeled as a posterior distribution of values, rather than as a single-value parameter.

\section{Model formulation}

The model in this study parameterized the transition rates between four classes of blister rust infection (susceptible, slightly infected, moderately infected, and dead) outlined at each site $k$ as: $S_{k} \rightarrow I_{k} \rightarrow M_{k} \rightarrow D_{k}$. The hierarchical Bayesian formulation (sensu Clark 2007) used in this analysis consists of three sub-models: the data model, the process model, and the parameter model (Fig. 3). The data model estimates the proportion of trees in each blister rust infection class at each site and year based on the field data, which describes the number of trees within each white pine blister rust infection class at each field census. In doing so, it accounts for sampling error by attributing different levels of confidence about infection rates that result from different sampling schemes. Observation errors associated with tree mis- 


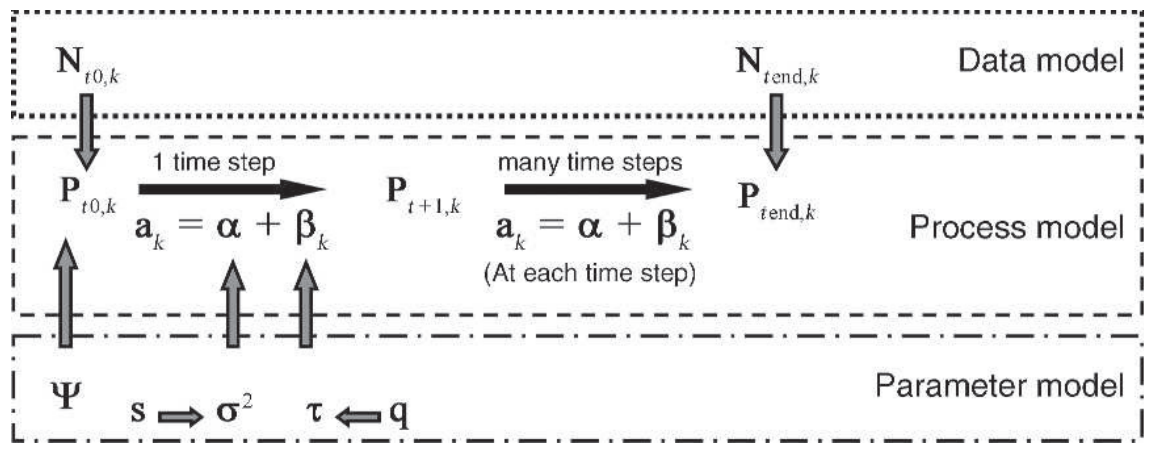

FIG. 3. The parameters within the hierarchical model are mapped according to the three levels: the data model, the process model, and the parameter model. During each time step $t$ for each site $k$, the three modeling levels interact to drive the process of infection between each of the four blister rust infection classes. Parameters are: $\mathbf{N}_{t, k}$, the data vector for tree count in each of the four classes (uninfected, slightly infected, moderately infected, dead) for year $t$ and site $k ; \mathbf{P}_{t, k}$ is the vector for the proportion of trees in each of the four classes in year $t$ and site $k$; $\boldsymbol{\Psi}$ is the prior on $\mathbf{P}_{t_{0}, k} ; \boldsymbol{\alpha}$ is the probability of transition between infection classes across all sites; $\boldsymbol{\sigma}^{2}$ is the variance on $\boldsymbol{\alpha} ; \boldsymbol{\beta}_{k}$ represents site-specific variation in transition probabilities for site $k ; \tau$ is the variance on $\boldsymbol{\beta}_{k} ; \mathbf{s}$ is the prior on $\boldsymbol{\sigma}^{2}$; and $\mathbf{q}$ is the prior on $\boldsymbol{\tau}$. Although most parameters within the model are fitted across all sites in the GYE, $\boldsymbol{\beta}_{k}$ varies at the site level.

classification are assumed to be small and not systematically different among data sets. The field data at each site $k$ in each year $t$ where data exists are modeled as a multinomial distribution:

$$
\mathbf{N}_{t, k}=\left[N_{S, t, k}, N_{I, t, k}, N_{M, t, k}, N_{D, t, k}\right] \sim \operatorname{Multinomial}\left(\mathbf{P}_{t, k}, \mathbf{N}_{t, k}\right)
$$

where the four elements of $\mathbf{N}_{t, k}$ represent the raw tree count data in the four infection classes from the aggregated data set, and as

$$
\mathbf{P}_{t, k}=\left[P_{S, t, k}, P_{I, t, k}, P_{M, t, k}, P_{D, t, k}\right]
$$

where the four elements of $\mathbf{P}_{t, k}$ represent the proportion of trees in each of the four blister rust infection classes. The multinomial distribution is a generalization of the binomial that describes the probability of observing tree counts given $N$ independent draws.

The process model describes the temporal dynamics of the proportion of trees in each blister rust infection class $\mathbf{P}_{t, k}$ as the infection progresses at each site $k$ at the annual time step, $t$. Disease progression is modeled as the transitions between the four classes of infection at an annual time step using a matrix model,

$$
\mathbf{P}_{t, k}=\mathbf{A}_{k} \mathbf{P}_{t-1, k}
$$

which indicates that the proportions of trees within the infection classes $\mathbf{P}_{t, k}$ at each site $k$ in each year $t$ are determined by the proportions in each infection class in the previous year multiplied by the site-specific transition matrix $\mathbf{A}_{k}$ :

$$
\mathbf{A}_{k}=\left(\begin{array}{cccc}
a_{S S, k} & 0 & 0 & 0 \\
a_{S I, k} & a_{I I, k} & 0 & 0 \\
0 & a_{I M, k} & a_{M M, k} & 0 \\
0 & 0 & a_{M D, k} & a_{D D, k}
\end{array}\right)
$$

where the elements of $\mathbf{A}_{k}$ represent the transition rates between the four blister rust infection classes. The elements of matrix $\mathbf{A}_{k}$ consist of both site-specific and global parameters. Within our analysis, we constrain
$a_{D D, k}$, the probability of remaining in the dead class, to 1 , since removal from the dead class through decomposition occurs on a much slower timescale than the scope of our current model. Similarly, we assume that seedling recruitment is negligible within the timescale of this model, and thus the total population size remains approximately constant. These constraints allow us to simplify the model, so that the column sums of $\mathbf{A}_{k}$ equal 1 , and we only need model the off-diagonal matrix elements:

$$
\mathbf{a}_{k}=\left[a_{S I, k}, a_{I M, k}, a_{M D, k}\right]
$$

where the elements of $\mathbf{a}_{k}$ are the transition rates between the four infection classes (susceptible, slightly infected, moderately infected, and dead). The transition rates $\mathbf{a}_{k}$ are calculated as

$$
\mathbf{a}_{k}=\alpha+\boldsymbol{\beta}_{k}+\varepsilon
$$

where $\alpha$ describes the global mean transition rate across all sites between each of the four blister rust infection classes, $\boldsymbol{\varepsilon}$ describes the variance on the mean transition process, and $\boldsymbol{\beta}_{k}$ describes a site-specific transition parameter. Within this analysis, $\boldsymbol{\alpha}$ is modeled as

$$
\boldsymbol{\alpha}=\left[\alpha_{S I}, \alpha_{I M}, \alpha_{M D}\right] \sim \mathcal{N}\left(a_{0}, V_{a}\right)
$$

where the elements of $\boldsymbol{\alpha}$ correspond to the transition rates $\mathbf{a}_{k}$. The elements of $\boldsymbol{\alpha}$ have a multivariate normal prior with mean $a_{0}$ and variance $V_{a}$. The process variance on $\boldsymbol{\alpha}$ is modeled as a multivariate normal distribution, with variance

$$
\begin{aligned}
\boldsymbol{\varepsilon} & =I \boldsymbol{\sigma}^{2} \sim \mathcal{N}\left(0, \boldsymbol{\sigma}^{2}\right) \\
\boldsymbol{\sigma}^{2} & =\left[\sigma_{S I}^{2}, \sigma_{I M}^{2}, \sigma_{M D}^{2}\right] \sim \operatorname{IG}\left(\mathbf{s}_{0}, \mathbf{s}_{1}\right)
\end{aligned}
$$

where $\boldsymbol{\sigma}^{2}$ includes the elements of a diagonal covariance matrix that represent the process variance on the transitions between the four classes across all sites, $\boldsymbol{\alpha}$. 
The elements of $\boldsymbol{\sigma}^{2}$ are assumed to have inverse gamma (IG) priors with parameters $\mathbf{s}_{0}$ and $\mathbf{s}_{1}$. Thus, $\boldsymbol{\alpha}$ is interpreted as the mean transition process between each of the four classes across all sites, and $\boldsymbol{\sigma}^{2}$ is the variation on that process. The parameter $\boldsymbol{\beta}_{k}$ describes the site-level variation in the rate of disease progression as

$$
\boldsymbol{\beta}_{k}=\left[\beta_{S I}, \beta_{I M}, \beta_{M D}\right] \sim \mathcal{N}\left(0, \tau^{2}\right)
$$

where $\boldsymbol{\beta}_{k}$ are the elements of a diagonal covariance matrix that represents variation within each site $k$ on the transitions between the four classes of white pine blister rust infection. $\boldsymbol{\beta}_{k}$ is modeled as a random site-specific influence on the transition rate chosen within our model from the multivariate normal distribution with mean 0 and variance $\tau^{2}$. Because $\mathbf{P}_{t, k}$ describes the change in the proportion of each infection class per year, alpha, beta, and epsilon all have units of $1 /$ year.

With these three parameters influencing the transition rates, the state transitions for each class of blister rust infection at each site $k$ in each year $t+1$ are given as

$$
\begin{aligned}
S_{t+1, k} & =-\left(\alpha_{S I}+\beta_{S I, k}\right) S_{t, k}+\varepsilon_{S I} \\
I_{t+1, k} & =\left(\alpha_{S I}+\beta_{S I, k}\right) S_{t, k}-\left(\alpha_{I M}+\beta_{I M, k}\right) I_{t, k}+\varepsilon_{S I}+\varepsilon_{I M} \\
M_{t+1, k} & =\left(\alpha_{I M}+\beta_{I M, k}\right) I_{t, k}-\left(\alpha_{M D}+\beta_{M D, k}\right) M_{t, k}+\varepsilon_{I M}+\varepsilon_{M D} \\
D_{t+1, k} & =\left(\alpha_{M D}+\beta_{M D, k}\right) M_{t, k}+\varepsilon_{M D} .
\end{aligned}
$$

As before, the proportions in each infection class in the ext time step, year $t$ at each site $k$, are determined by the proportion of trees in each infection class at the current time step multiplied by the transition rates, composed of parameters $\boldsymbol{\alpha}, \boldsymbol{\beta}_{k}$, and, implicitly, $\boldsymbol{\sigma}^{2}$. The three parameters that contribute to variation in the process of blister rust infection are outlined schematically as Fig. 4a, and the improved performance of the model with the inclusion of all three sources of process variability is plotted for one example site as Fig. 4b. Note that, although the process is computed at an annual time step, the parameters are assumed to be time invariant.

Because the population at each site $k, \mathbf{P}_{t, k}$, is not evaluated annually within the aggregated data set every year $t$, the posterior estimates of $\mathbf{P}_{t, k}$, which are a latent, unobserved quantity in the model, are constrained by the population state at both the previous and following time steps, $\mathbf{P}_{t-1, k}$ and $\mathbf{P}_{t+1, k}$, according to the process model:

$$
\mathbf{P}_{t, k} \sim \mathcal{N}\left(\mathbf{P}_{t+1, k} \mid \mathbf{P}_{t, k}, \boldsymbol{\sigma}^{2}\right) \cdot \mathcal{N}\left(\mathbf{P}_{t, k} \mid \mathbf{P}_{t-1, k}, \boldsymbol{\sigma}^{2}\right) .
$$

Model formulation is simplified by the fact that each plot has only been re-censused once, and thus the first and last census were used as the starting and ending points when modeling each site $k$. Modeling this process constrained by the two field censuses introduces two additional cases in the calculation of the process of blister rust infection, where the first and last model steps depend on the field data $\mathbf{N}_{t, k}$ :

$$
\begin{aligned}
& \mathbf{P}_{t_{0}, k} \sim \mathcal{N}\left(\mathbf{P}_{t_{0}+1, k} \mid \mathbf{P}_{t_{0}, k}, \boldsymbol{\sigma}^{2}\right) \cdot \operatorname{Multinomial}\left(\mathbf{N}_{t_{0}, k} \mid \mathbf{P}_{t_{0}, k}\right) \\
& \cdot \operatorname{Dirichlet}\left(\mathbf{P}_{t_{0}, k} \mid \Psi\right) \\
& \mathbf{P}_{t_{\text {end }}, k} \sim \mathcal{N}\left(\mathbf{P}_{t_{\text {end }}, k} \mid \mathbf{P}_{t_{\text {end }}, k}, \boldsymbol{\sigma}^{2}\right) \cdot \operatorname{Multinomial}\left(\mathbf{N}_{t_{\text {end }}, k} \mid \mathbf{P}_{t_{\text {end }}, k}\right)
\end{aligned}
$$

where $\mathbf{N}_{t_{0}, k}$ and $\mathbf{P}_{t_{0}, k}$ represent, respectively, the number and modeled proportion of trees in each of the infection classes at the first field census, and $\mathbf{N}_{t_{\text {end }}, k}$ and $\mathbf{P}_{t_{\text {end }}, k}$ represent, respectively, the number and proportion of trees in each infection class at the last field census. For the initial time step there is a prior on the initial model state, modeled by the Dirichlet distribution with shape parameters $\boldsymbol{\Psi}$ equal to 0.9 , to represent a very low probability of initial infection at all sites.

\section{Metapopulation formulation}

The model just presented describes the case of local population dynamics at each site with a static infection rate. To evaluate the dynamics of white pine blister rust infection at both the local level within sites and the global level among all sites, we created four dynamic models. Two of the models only contain local infection parameters, and the other two models take on a metapopulation format, in which blister rust infection occurs both within sites and among sites. Thus, the differences between the models arise in the density dependence of local and global infection. Although the simplest model considers no density-dependent white pine blister rust infection, the other models consider combinations of local and global density-dependent infection in order to evaluate the transition dynamics of white pine blister rust infection. The differences among the models arise in the terms describing the transition between the susceptible and slightly infected class.

Model 1.-Static local infection:

$$
\begin{aligned}
& S_{t+1, k}=-\left(\alpha_{S I}+\beta_{S I, k}\right) S_{t, k} \\
& I_{t+1, k}=\left(\alpha_{S I}+\beta_{S I, k}\right) S_{t, k}-\left(\alpha_{I M}+\beta_{I M, k}\right) I_{t, k} .
\end{aligned}
$$

Model 2.-Dynamic local infection:

$$
\begin{aligned}
& S_{t+1, k}=-\left(\alpha_{S I}+\beta_{S I, k}\right) S_{t, k}\left(I_{t, k}+M_{t, k}\right) \\
& I_{t+1, k}=\left(\alpha_{S I}+\beta_{S I, k}\right) S_{t, k}\left(I_{t, k}+M_{t, k}\right)-\left(\alpha_{I M}+\beta_{I M, k}\right) I_{t, k} .
\end{aligned}
$$

Model 3.-Dynamic local infection, static global infection:

$$
\begin{aligned}
& S_{t+1, k}=-\left(\alpha_{S I}+\beta_{S I, k}\right) S_{t, k}\left(I_{t, k}+M_{t, k}\right)-b \\
& I_{t+1, k}=\left(\alpha_{S I}+\beta_{S I, k}\right) S_{t, k}\left(I_{t, k}+M_{t, k}\right)-\left(\alpha_{I M}+\beta_{I M, k}\right) I_{t, k}+b .
\end{aligned}
$$

Model 4.-Dynamic local infection, dynamic global infection:

$$
S_{t+1, k}=-\left(\alpha_{S I}+\beta_{S I, k}\right) S_{t, k}\left(I_{t, k}+M_{t, k}\right)-g\left(I_{t, g}+M_{t, g}\right)
$$


a

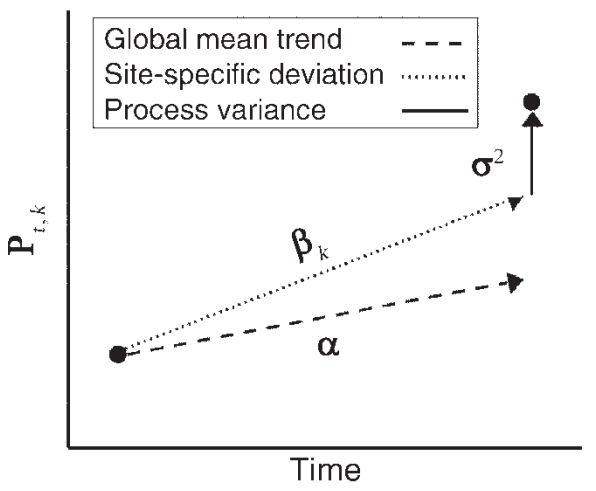

b

$-\alpha$ only

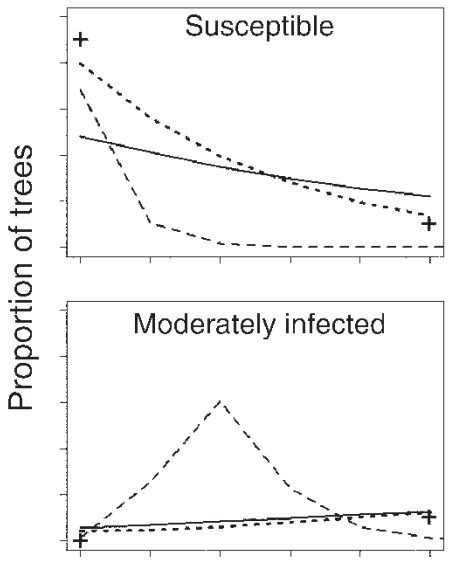

Year $-\alpha$ and $\sigma^{2} \quad \cdots, \alpha, \sigma^{2}, \quad+$ Data
and $\beta_{k}$
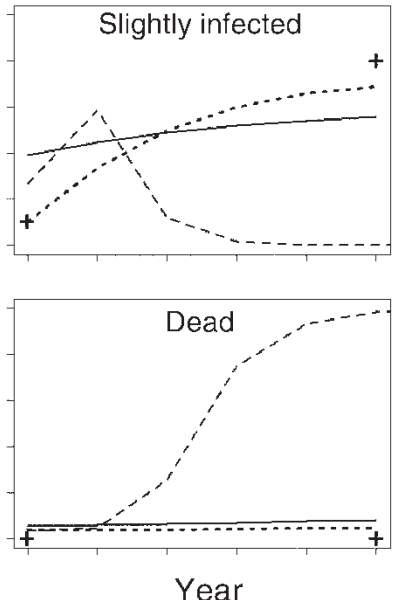

FIG. 4. (a) Effects of the global transition rates $\boldsymbol{\alpha}$, the variance $\boldsymbol{\sigma}^{2}$ on the global transition rates, and the site-specific effects $\boldsymbol{\beta}_{k}$ within the model on $\mathbf{P}_{t, k}$. (b) Conceptual modeled output for the proportion of trees in each category, with all three parameters at one example site plotted against field data for that site. Inclusion of all three parameters (dotted line) clearly improves the overall model performance.

$$
\begin{aligned}
I_{t+1, k}= & \left(\alpha_{S I}+\beta_{S I, k}\right) S_{t, k}\left(I_{t, k}+M_{t, k}\right)-\left(\alpha_{I M}+\beta_{I M, k}\right) I_{t, k} \\
& +g\left(I_{t, g}+M_{t, g}\right)
\end{aligned}
$$

where indexing is as before across sites $k$ at an annual time step $t$, except for Models 3 and 4, which introduce new parameters, $b$, which represents a static background of blister rust spore influx to the ecosystem, and $g$, a global rate of infection that is proportional to the fraction of the landscape in the infective (and therefore white pine blister rust spore-producing) classes across all sites $\left(I_{t, g}+M_{t, g}\right)$. The variable $\left(I_{t, g}+M_{t, g}\right)$ is obtained within the model as the sample mean across all sites by integrating across the posterior uncertainty for all sites at each year $t$. In the models that represent dynamic local infection (Models 2, 3, and 4), we make the transition between the susceptible and infected classes at each site $k$ and time step $t$ dependent on the proportion within the infective classes (slightly infected and moderately infected) at that site $k\left(I_{t, k}+M_{t, k}\right)$. Note that with our parameter formulation, the combination of static local and either static or dynamic global infection is impossible, because when local infection does not vary with the proportion of locally infected trees, the parameter that represents the global mean transitions, $\boldsymbol{\alpha}$, and the parameters that represent the global infection rates, $b$ and $g$, become redundant and the model collapses to the simpler model with static local infection and no global infection. We emphasize that the $\boldsymbol{\alpha}$ and $\boldsymbol{\beta}_{k}$ parameters assume slightly different meanings through the different dynamic formulations, but we have maintained this notation among all four models for the sake of clarity. All models were implemented in R, and the annotated code used to run the models is included in the Supplement.

\section{Model fitting}

The models were implemented with the aggregated data set through a Markov chain Monte Carlo (MCMC) scheme with 100000 iterations per model that was computed four different times per model to assure convergence of all parameters. Convergence of the parameters within the four models was determined by visually inspecting the parameter values plotted against the MCMC iterations to ensure both parameter mixing and parameter convergence to a stable mean over the 100000 iterations within the MCMC routine.

The fit of the four dynamic models is compared using the predictive loss criterion, which evaluates model performance by minimizing the predictive loss of the posterior distribution of the parameters (Gelfand and Ghosh 1998, Clark 2007). We chose the predictive loss criterion over other metrics of Bayesian model selection because it emphasizes the performance of prediction into the future, which matched with our goal of predicting the future progress of blister rust at different sites throughout the GYE.

The predictive loss value $D_{m}$ for each model $m$ is calculated as the sum of two terms: $G_{m}+P_{m}$. $G_{m}$ is the error sum of squares, which is the cost for selecting the wrong model:

$$
G_{m}=\sum_{i=1}^{n}\left(E\left[y_{i} \mid \mathbf{y}\right]-y_{i}\right)^{2}
$$

where $\mathbf{y}$ is the matrix of $y$ values. $P_{m}$ is the penalty term, which is the predictive variance:

$$
P_{m}=\sum_{i=1}^{n} \operatorname{var}\left[y_{i} \mid \mathbf{y}\right] .
$$


TABle 2. Predictive loss model scores, used to assess model fitness in Bayesian statistics, for each of the four dynamical models.

\begin{tabular}{lc}
\hline \hline \multicolumn{1}{c}{ Model } & Predictive loss score \\
\hline 1) Static local infection, no global infection & 48800.54 \\
2) Dynamic local infection, no global infection & 29834.29 \\
3) Dynamic local infection, static global infection & 33497.91 \\
4) Dynamic local infection, dynamic global infection & 28463.08 \\
\hline
\end{tabular}

Notes: A lower score indicates a better-fit model, and thus Model 4 is the best fit for the data set, closely followed by Models 2 and 3. All other models significantly outperformed Model 1, which is the only model that does not account for density-dependent infection within sites.

The model with the lowest value of $D_{m}$ is considered the best fit (Clark 2007). The units of $D_{m}$ are absolute values because both $G_{m}$ and $P_{m}$ scale with sample size, and therefore can only be accurately compared between models fitted to the same data set.

\section{RESULTS}

Each of the four dynamic blister rust infection models was parameterized with the aggregated data set composed of 121 blister rust infection sites that span 19682008, and the model parameterizations for each site are included in the Appendix. The predictive loss criterion was computed for each of the four model outputs, and the values for the four models are included as Table 2. A comparison of the predictive loss scores between the four models clearly demonstrates that the three models describing dynamic local infection (Models 2, 3, and 4) outperform the model with static infection (Model 1). Model 4, which incorporates both dynamic local and global infection, is the best-fit model according to the predictive loss criterion. However, we also considered the predictive loss score of Model 2, the model with dynamic local infection and no global infection, as close enough to that of Model 4 to also merit interpretation.

The model outputs for each site can be visually compared to further evaluate the relative performance of the four models. The output from six representative sites, selected to compare the sensitivity of model fitting, is included as Fig. 5. We chose these six sites to highlight model performance regarding: (1) the time span of data included in the model, (2) the amount of initial infection during the first field census, and (3) the change in the proportion of infected trees between the first and last field censuses.

In Fig. 5, site A represents a site with a data set that spans the largest time frame in our model, 1968-2008, and site B spans the smallest time frame of data, 20042007. Comparing sites A and B, over the large timescale at site A, models 2 and 4 clearly outperform models 1 and 3 , following the conclusions of the predictive loss criterion for model performance. However, the four model outputs in Site B demonstrate that over short timescales that result in very small changes in the number of infected trees between field censuses, the four model outputs are generally indistinguishable. This comparison highlights the fact that the effects of density dependence in white pine blister rust infection manifest over longer timescales that exceed the span of 20042007.

In Fig. 5, sites $\mathrm{C}$ and $\mathrm{D}$ were selected to highlight model performance within sites that were either uninfected (site C) or had very low amounts of initial infection (site D). Analyzing the model outputs at these sites shows that over a moderate time frame of 13-14 years in which the blister rust infection levels were either low or nonexistent in the first census, Models 2 and 4, and in some cases also Model 3, accurately captured the infection dynamics. It is evident from Fig. 5, sites $\mathrm{C}$ and $\mathrm{D}$, that Model 1, which does not consider dynamic local infection, was unable to accurately represent these sites with low or nonexistent levels of initial infection. The results at sites $\mathrm{C}$ and $\mathrm{D}$ also support the conclusion of the predictive loss model evaluation, where Models 2 and 4 performed best, followed by Model 3, and Model 1 scored particularly low.

In Fig. 5, sites $\mathrm{E}$ and $\mathrm{F}$ were selected to compare model performance at sites that showed a dramatic rise in the level of infection between the first and last field censuses. The comparison in model performance between these sites demonstrates that at sites with moderate time spans and a dramatic rise in the rate of blister rust infection during the time period, the four models generally perform equally well. During a dramatic rise in infection in a relatively small time frame (2001-2007 at site E and 1995-2008 in site F), the site-specific parameter $\boldsymbol{\beta}_{k}$ may dominate the infection dynamic compared with other factors, for example density-dependence. All four models contain the parameter $\boldsymbol{\beta}_{k}$, which at these sites probably dominates the modeled site-level infection; this may explain the relatively equal performance of all models at these sites with dramatic increases in blister rust infection.

Fig. 6a shows the transition dynamics between the four infection classes averaged across all sites in the years between which data are present for the time period 1968-2008, and Fig. 6b shows the transition dynamics from 1968-2008 averaged across only the 11 sites sampled at the earliest (first sampling date 1968-1972) time points in the analysis. Consistent with the predictive loss scores (Table 2), Models 2 and 4 strongly outperform Model 1, demonstrating the importance of considering local density-dependent infection within the model. Model 3 also generally performs well, but with a greater tendency to over-predict the proportion of 


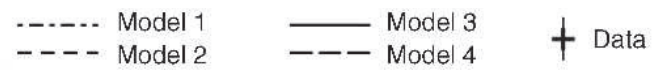

Site A
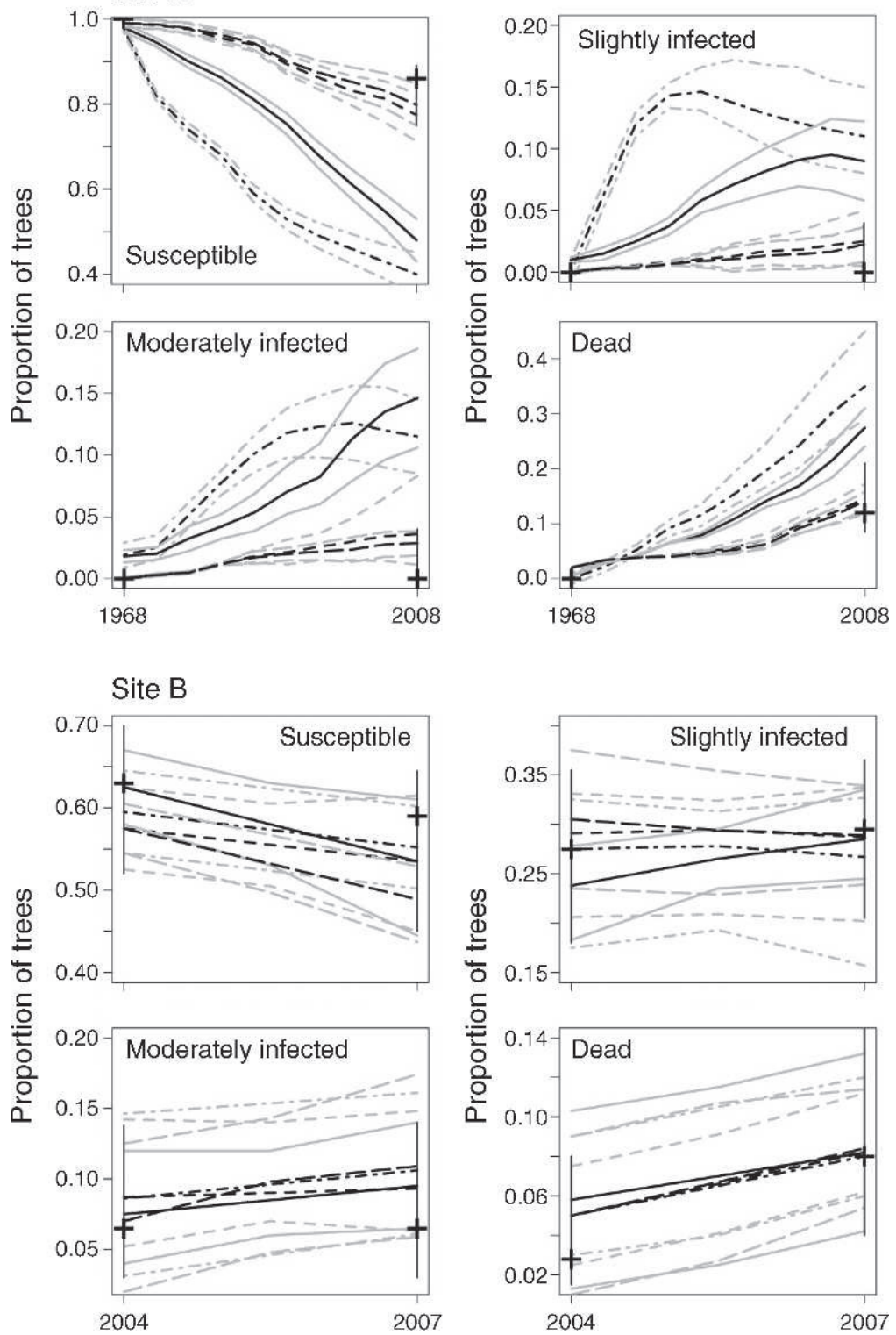

FIG. 5. Output of the models for the proportion of trees in each category at six different field plots (sites A-F), chosen to be representative of variation within the modeled data set. Outputs at these different field sites show the median posterior output for each of the four dynamic models (black lines), with $95 \%$ CIs from the posterior distribution for each model (gray lines). For the endpoint years, the field data are plotted as the mean proportion in each field class (black cross), with the $95 \%$ CI from the posterior distribution shown as the vertical solid line.

uninfected individuals and to under-predict the proportions of infected individuals compared with Models 2 and 4. In Fig. 6, the jump in the level of infection in the mid-1990s reflects the portion of our model that is data rich, because most data sets fall within the period 19952008.

\section{Model predictions}

As demonstrated by the sharp change during the past decade in Fig. 6, models 2, 3, and 4 all indicate a rapidly accelerating transition rate between the uninfected and infected classes. Although our model is computed empirically according to the past activity of white pine blister rust at specific field sites, this implies that if ecological conditions remain similar to those during the time frame for which the model was parameterized, blister rust infestation will continue to accelerate along this trajectory into the future. Fig. 7 plots the future predicted levels of blister rust infestation for the ecosystem over the next 10 years based on the parameter estimates for Models 2, 3, and 4 computed during the 1968-2008 time period (Model 1 was excluded from this 

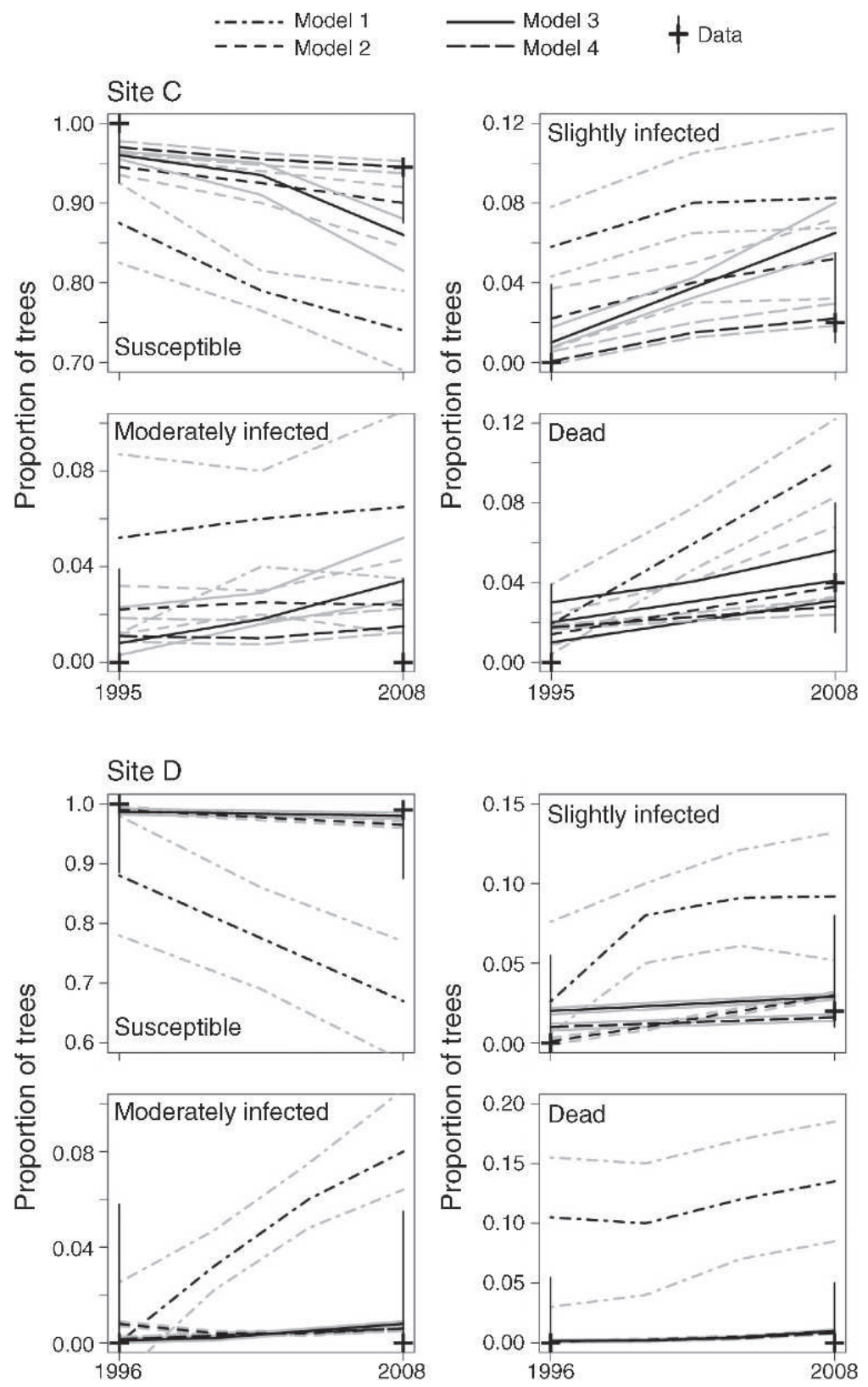

FIg. 5. Continued.

analysis due to its poor performance as determined by its high predictive loss score). In the future ecosystem predictions in Fig. 7, the three models again perform similarly, and only differ slightly in the rate of transition between the uninfected and slightly uninfected class. Model 4 exhibits the fastest acceleration to an average of $90 \%$ infection rate at all sites by the year 2013. This rapid decline compared with the other two models is due to the density dependence in the rate of global infection within Model 4, which does not exist in the other two models. Model 2 predicts an increase to $90 \%$ ecosystem infection in 2026 and Model 3 predicts the same for 2033. Models 2 and 3 exhibit a slower decline to $90 \%$ global infection at all sites due to the dependence on only local infection in Model 2, and the constant rate of global infection in Model 3.

In our model formulations, we included a site-specific random effect, the $\boldsymbol{\beta}_{k}$ parameter, in order to capture environmental heterogeneity between sites in the blister rust infection process. The inclusion of $\boldsymbol{\beta}_{k}$ significantly improves model performance (Fig. 4). Within our model formulation, the $\boldsymbol{\beta}_{k}$ site effects parameter is modeled as a random effect for each site, which we hypothesized might correlate with sources of environmental heterogeneity that would help to explain differences in the rate of blister rust infection at individual sites. Although the 

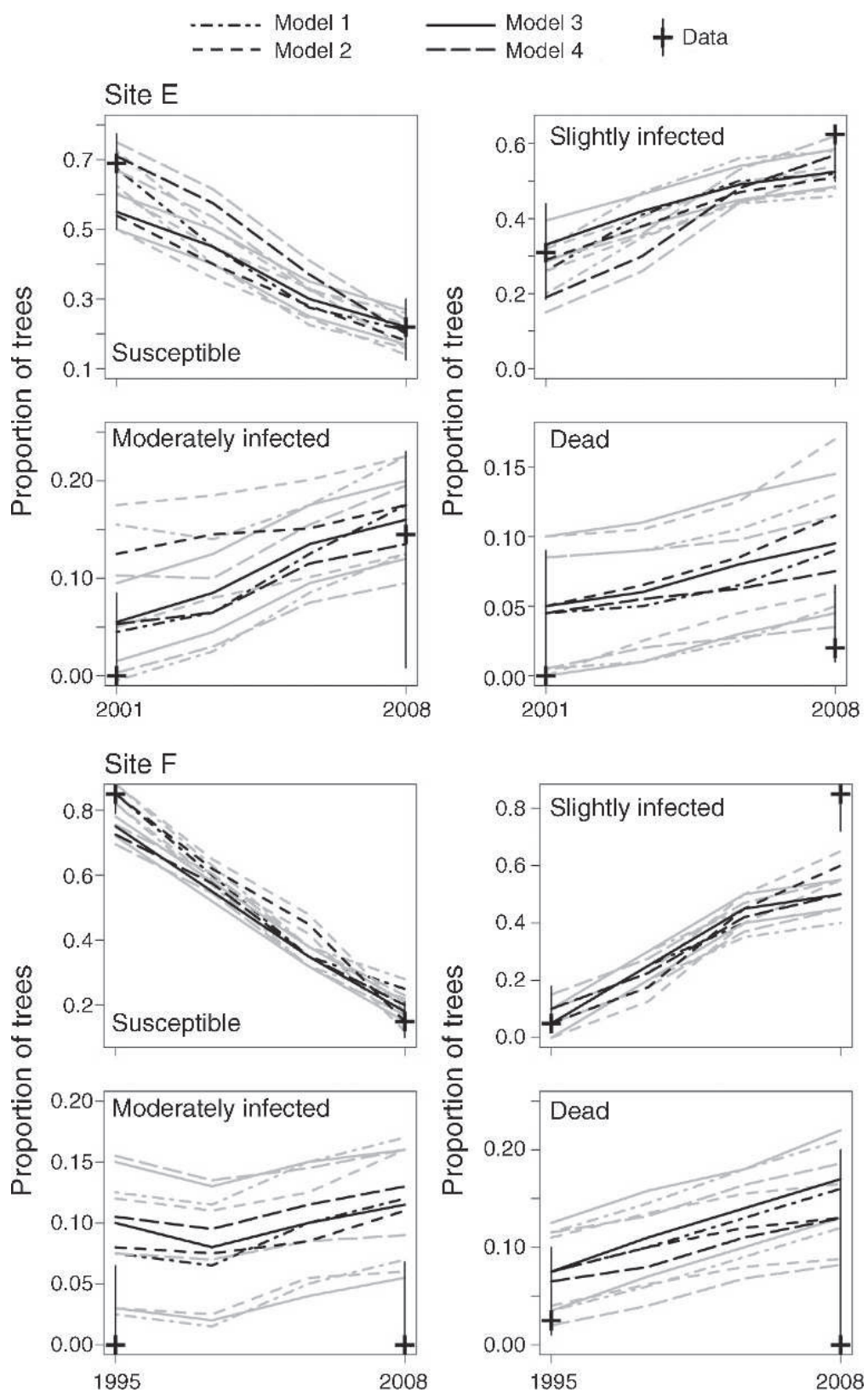

FIG. 5. Continued.

analysis indicates that model performance is clearly enhanced by the inclusion of the $\boldsymbol{\beta}_{k}$ parameter, we were not able to identify an obvious environmental driver for this parameter. A preliminary analysis showed that differences in $\boldsymbol{\beta}_{k}$ between sites were not related to slope, aspect, or elevation. Although early studies demonstrated a link between local microclimate environmental conditions and blister rust prevalence (van Arsdel et al. 1956), the lack of significant correlation with site variables as in our study was also exhibited in whitebark pine trees infected with white pine blister rust in British Columbia (Campbell and Antos 2000). We also found no significant spatial autocorrelation in $\boldsymbol{\beta}_{k}$. The lack of spatial or environmental correlation may indicate that the blister rust spores within the ecosystem are so ubiquitous that local environmental conditions are insignificant in controlling the spread of the disease, and therefore only infective tree density is significant when considering the infection dynamics.

Table 3 outlines the computed model parameters $\boldsymbol{\alpha}$, $\boldsymbol{\sigma}^{2}, b$, and $g$ (when applicable) for all four models. The values for the hierarchical variance $\sigma^{2}$ on the process of transition between the four infection classes converged to nearly the same values for Models 2, 3, and 4, demonstrating that the amount of process error within the models is well constrained. Ecologically, this indicates that between the three dynamic infection scenarios, the amount of error in our understanding of 


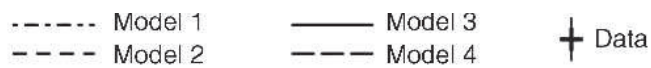

a) Ecosystem-level infestation for all sites
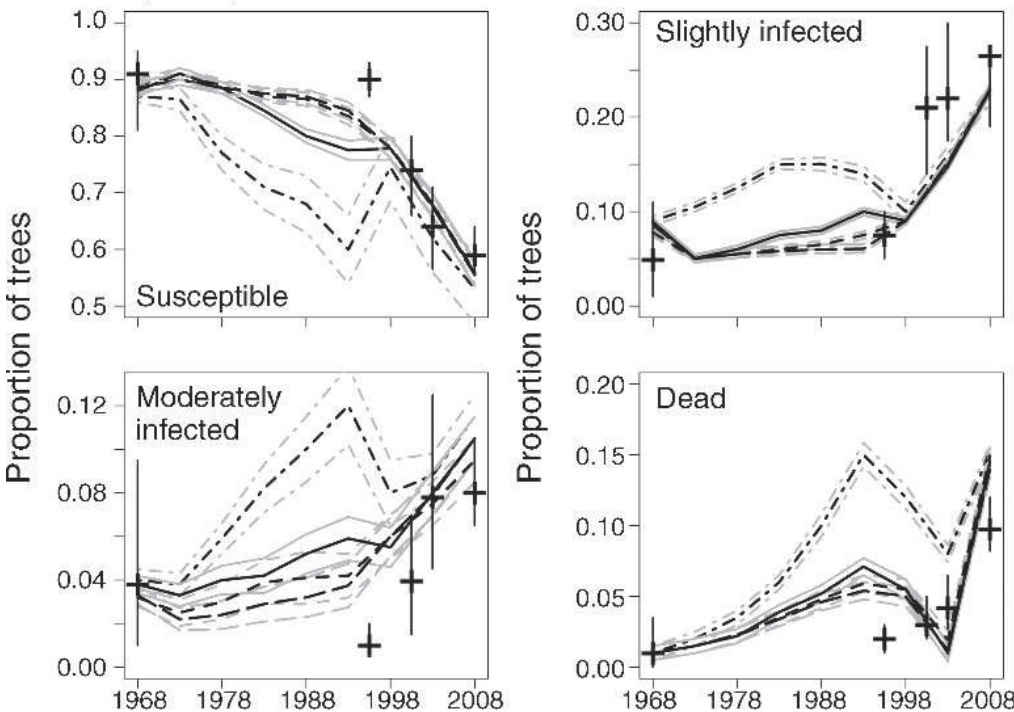

b) Infestation for 11 sites first surveyed 1968-1972
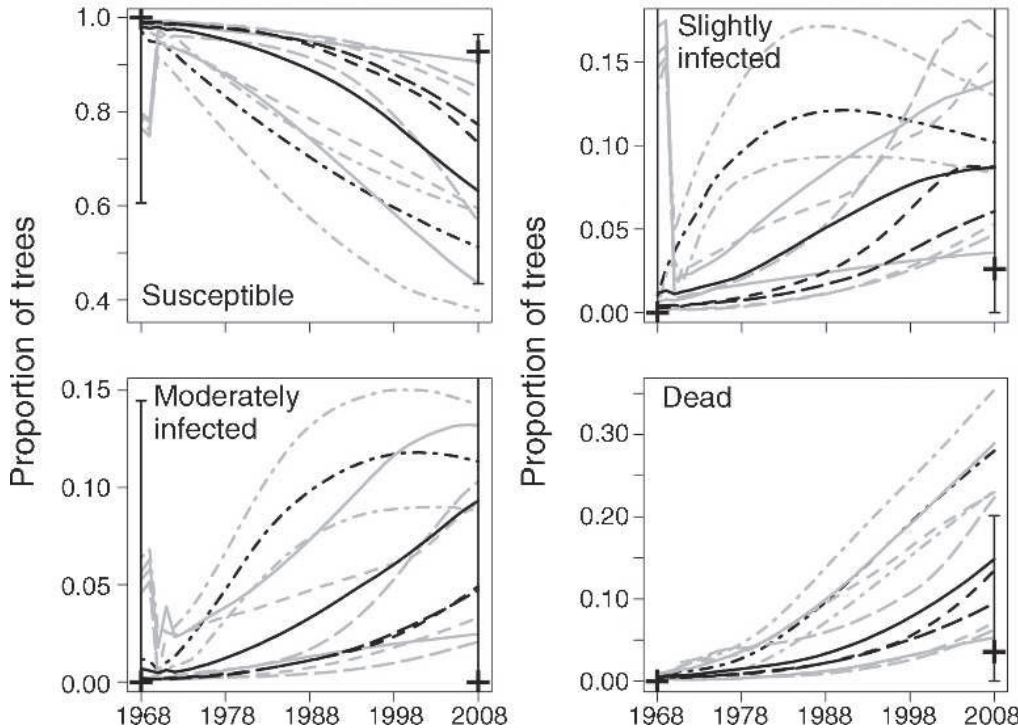

FIG. 6. (a) Ecosystem-wide level of blister rust infestation for the time period 1968-2008, averaged from the Markov chain Monte Carlo output aggregated for all sites, plotted as the median of the posterior distribution (black lines) with $95 \%$ CIs (gray lines). The data points (black crosses) represent the median infection from the field surveys within each year, with the posterior $95 \%$ CIs from the model shown as a vertical line. Model 4, which contains terms that describe both site-level and ecosystem-wide infective tree density dependence, performs the best. However, Models 2 and 3 also perform relatively well compared with Model 1, which indicates that site-level density dependence may be the most important factor in determining white pine blister rust infection dynamics. (b) Level of blister rust infestation for 1968-2008 shown only for the 11 sites first sampled in 1968-1972, averaged from the Markov chain Monte Carlo output for these 11 sites, plotted as the median of the posterior distribution (black lines), with $95 \%$ CI (gray lines). All models except Model 1 perform relatively well at capturing the blister rust infection rates during this relatively long time period.

the infection transition process in our model is relatively small and constant among the three models. The values for the hierarchical variance $\tau^{2}$ on the site effects $\boldsymbol{\beta}_{k}$ varied among all models, indicating that the relative contribution of the site effects parameter varied from model to model. The variance on the site effect, $\tau^{2}$, is highest for Model 4, the best-fit model. Because the site effects $\boldsymbol{\beta}_{k}$ are selected from a random normal distribution, a higher value of variance indicates that the model parameterization expresses higher site-level environmental variability $\boldsymbol{\beta}_{k}$ among sites. Because Model 4 was the best fit, this indicates that variability among sites is 


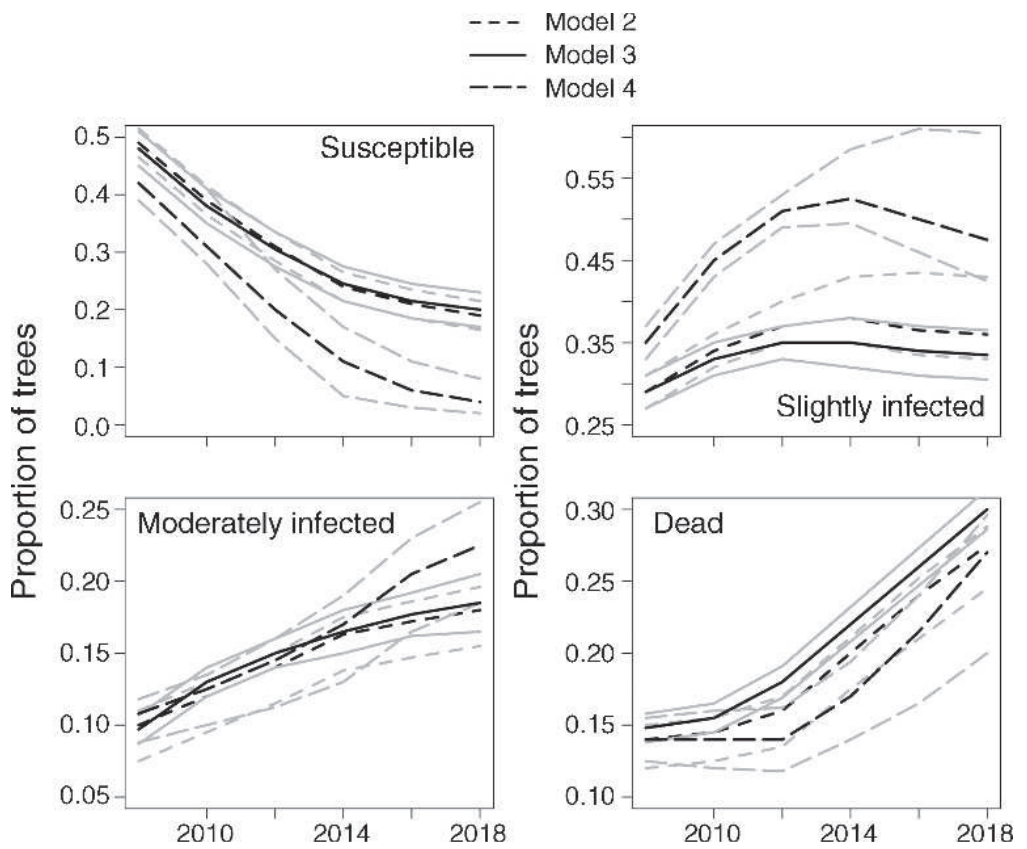

FIG. 7. Globally averaged projections (aggregated for all sites) for the future level of blister rust in the Greater Yellowstone Ecosystem plotted as the median of the posterior distribution (solid lines) with 95\% CIs (dotted lines). The projections differ in the rates of increase for each dynamic model formulation. Although our current model is parameterized with data that reflect conditions during the last 40 years, changes in the environment due to climate change and pathogen or pest interactions might also influence the rate of white pine blister rust progression.

relatively more important than ecosystem-wide process error variance $\sigma^{2}$ in determining the overall transition rates.

\section{Discussion}

By comparing the performance of the four model formulations, we can compare different hypotheses about the dynamics of blister rust infection in whitebark pine at the ecosystem scale. The improved performance of all models that included local density dependence in infection rate (Models 2, 3, and 4) compared to the density-independent model (Model 1) indicates that blister rust infestation in whitebark pine spreads through mechanisms that are dependent on the amount of local infestation, despite the fact that blister rust does not spread directly from tree to tree. The importance of the local density dependence term indicates that the most likely pathway for the spread of blister rust spores is dispersion from whitebark pines to local alternate hosts and back to local whitebark pines within a single

TABLE 3. Posterior distribution of the median and upper and lower $95 \%$ CI for the ecosystem-wide parameters from the four different dynamic infection models.

\begin{tabular}{lcccccccccccc}
\hline \hline $\begin{array}{c}\text { Model and } \\
\text { statistic }\end{array}$ & $\boldsymbol{\alpha}_{\mathrm{SI}}$ & $\boldsymbol{\alpha}_{\mathrm{IM}}$ & $\boldsymbol{\alpha}_{\mathrm{MD}}$ & $\boldsymbol{\sigma}_{\mathrm{S}}^{2}$ & $\boldsymbol{\sigma}_{\mathrm{I}}^{2}$ & $\boldsymbol{\sigma}_{\mathrm{M}}^{2}$ & $\boldsymbol{\sigma}_{\mathrm{D}}^{2}$ & $\tau_{\mathrm{SI}}$ & $\tau_{\mathrm{IM}}$ & $\tau_{\mathrm{MD}}$ & $b$ & $g$ \\
\hline 1) Lower CI & 0.0315 & 0.0718 & 0.0842 & 0.0431 & 0.0419 & 0.031 & 0.0374 & 0.8345 & 0.3965 & 0.2175 & $\mathrm{~N} / \mathrm{A}$ & $\mathrm{N} / \mathrm{A}$ \\
Median & 0.0288 & 0.0792 & 0.1042 & 0.0627 & 0.0538 & 0.0376 & 0.0438 & 1.176 & 0.5252 & 0.3952 & $\mathrm{~N} / \mathrm{A}$ & $\mathrm{N} / \mathrm{A}$ \\
Upper CI & 0.0237 & 0.0923 & 0.1123 & 0.0743 & 0.0597 & 0.0418 & 0.0497 & 1.487 & 0.6459 & 0.5218 & $\mathrm{~N} / \mathrm{A}$ & $\mathrm{N} / \mathrm{A}$ \\
2) Lower CI & 0.1187 & 0.0679 & 0.0786 & 0.0172 & 0.0229 & 0.0282 & 0.0249 & 1.089 & 0.6687 & 0.4349 & $\mathrm{~N} / \mathrm{A}$ & $\mathrm{N} / \mathrm{A}$ \\
Median & 0.1314 & 0.0835 & 0.1043 & 0.0182 & 0.0244 & 0.0301 & 0.0264 & 1.312 & 0.8452 & 0.6208 & $\mathrm{~N} / \mathrm{A}$ & $\mathrm{N} / \mathrm{A}$ \\
Upper CI & 0.1526 & 0.0983 & 0.1202 & 0.0194 & 0.0263 & 0.0343 & 0.0288 & 1.621 & 1.023 & 0.8852 & $\mathrm{~N} / \mathrm{A}$ & $\mathrm{N} / \mathrm{A}$ \\
3) Lower CI & 0.0785 & 0.0721 & 0.1186 & 0.0172 & 0.0219 & 0.0277 & 0.0254 & 1.293 & 0.4786 & 0.3734 & 0.00134 & $\mathrm{~N} / \mathrm{A}$ \\
Median & 0.0978 & 0.0824 & 0.1051 & 0.0181 & 0.0235 & 0.0301 & 0.0279 & 1.576 & 0.633 & 0.4928 & 0.00159 & $\mathrm{~N} / \mathrm{A}$ \\
Upper CI & 0.1372 & 0.0938 & 0.0841 & 0.0192 & 0.0252 & 0.0338 & 0.0302 & 2.058 & 0.8872 & 0.6892 & 0.00177 & $\mathrm{~N} / \mathrm{A}$ \\
4) Lower CI & 0.1247 & 0.0832 & 0.0897 & 0.0175 & 0.0228 & 0.0287 & 0.0242 & 0.9472 & 0.7302 & 0.6587 & N/A & 0.0000199 \\
Median & 0.1493 & 0.0916 & 0.1068 & 0.0185 & 0.0243 & 0.0317 & 0.0264 & 1.169 & 0.9182 & 0.8027 & N/A & 0.0000476 \\
Upper CI & 0.1812 & 0.1043 & 0.1352 & 0.0203 & 0.0279 & 0.0362 & 0.0299 & 1.401 & 1.1103 & 1.1 & N/A & 0.0000609 \\
\end{tabular}

Notes: Each of the four models was computed as 100000 Markov chain Monte Carlo simulations and was averaged over four runs for each model. N/A means not applicable. Terms: $\alpha$ is the mean transition rate between classes, where SI is the transition from susceptible to slightly infected, IM is the transition from slightly to moderately infected, and MD is the transition from moderately infected to dead; $\boldsymbol{\sigma}^{2}$ is the variance for the proportion of trees in each infection class where $\mathrm{S}$ is the susceptible class, $\mathrm{I}$ is slightly infected, $\mathrm{M}$ is moderately infected, and $\mathrm{D}$ is the dead class; $\tau$ is the variance for the site-specific effects $\boldsymbol{\beta}_{k}$ on the transition rates; $b$ is the static background infection rate for Model 3; and $g$ is the density-dependent background infection rate for Model 4. 
growing season. One possible explanation for this local density dependence is that the alternate hosts for the spread of white pine blister rust are widely dispersed through the landscape, so that virtually all whitebark pine populations are situated close enough to a local patch of alternate hosts in order to complete the spore life cycle within a single growing season. However, our results reached no clear conclusion regarding the possibility of regional-scale density dependence in white pine blister rust infection rates, and further studies could tease apart the limits of the spatial scales at which this local infection mechanism operates.

The fitted parameter values for the transition rates between the four stages of white pine blister rust infection can be transformed into mean residence times for each infection stage. Using the parameters from Model 4, the best-fit model, the mean residence times across all sites, $\boldsymbol{\sigma}$, indicate that whitebark pine trees in these sites take, on average, 6.7 years to transition from uninfected to infected, 10.9 years to transition from infected to moderately infected, and 9.4 years to transition from infected to dead. This is the first known field study of the rate of blister rust progression in highaltitude whitebark pine, and serves as an informative temporal parameter for forest managers within the GYE. Identifying the rate of progression of white pine blister rust will help to inform management decisions about transplanting genetically resistant whitebark pine within the GYE, which is considered a critical management solution to help buffer the negative impacts of white pine blister rust (Sniezko et al. 2004). The residence times of the slightly infected and moderately infected stages indicate that, on average in our data set, trees live for $\sim 20$ years with white pine blister rust infection, a longer infection period than other lower elevation pine species, such as sugar pine and western white pine infected by blister rust (Smith and Hoffman 2000). This indicates a temporal window in which heavily infected sites can be identified, monitored, and possibly replanted with resistant whitebark pine, while monitoring the disease progression of infected trees within the site.

Our current metapopulation model predicts well at individual sites, but we are currently limited when extending our model to one where space is treated explicitly, due to the lack of spatial environmental drivers found within our analysis of the site effects parameter $\boldsymbol{\beta}_{k}$. Although the $\boldsymbol{\beta}_{k}$ site effects were determined to be a statistically significant parameter to include within the model, they may represent a more complicated process, such as genetic resistance to infection within sites. Limiting our model to one that is spatially implicit presents some challenges to the interpretation of blister rust dynamics in the GYE, particularly when considering the spread of blister rust into areas where no data exist. In this modeling analysis, we treat space implicitly due to uncertainty in the initial conditions of blister rust distribution throughout the landscape, patchy infestation at a small scale, and lack of spatial autocorrelation in the infection data. The inclusion of additional data types would help to develop a spatially explicit model of disease spread. Although expensive to obtain and restricted to the analysis of current levels of white pine blister rust, data from hyperspectral airborne remote-sensing platforms show promise for categorizing the white pine blister rust infection through the landscape, and would help to constrain the spatial progression of the infection (Hatala et al. 2010). Modeling the progression of white pine blister rust infection in the future would also be improved by a data set that tracks the fate of tagged individual trees, particularly in areas with currently uninfected whitebark pine. This strategy might also help to identify genetic populations of whitebark pine that are resistant to white pine blister rust.

Future work could also improve predictions by changing the assumptions of the matrix model. Additional studies on whitebark pine dynamics within this ecosystem could support the addition of a seedling recruitment term to more accurately portray the population dynamics. Additionally, our model currently predicts some small probability of infection for every time step from the multinomial distribution used to model $\mathbf{P}_{t, k}$. The basic formulation presented within this analysis could be changed to a mixture model, with some probability of presence/absence of the disease. Although this analysis demonstrates that the current model performs reasonably well for this baseline temporal analysis of white pine blister rust infection dynamics, additional future data could improve the predictions of this model by adding complexity while decreasing parameter uncertainty.

The predictions of Model 4, the best-fit model, suggest a $90 \%$ average infection rate across all sites by the year 2013, and Models 2 and 3, also fit relatively well to the data, calculate a $90 \%$ infection level by the years 2026 and 2033. This indicates that blister rust will continue to spread within whitebark pine in 10-20 years to a level at which nearly all trees at the sites will be impacted. Because the model developed within this analysis is parameterized based on current environmental conditions, we emphasize that the transition rates between infection classes might change due to future conditions and biotic feedbacks. Feedbacks due to future climate change could have large impacts on whitebark pine dynamics, particularly in interactions with other pests and pathogens such as the mountain pine beetle, recently shown to preferentially attack whitebark pine trees already infected with blister rust (Six and Adams 2007). Climate change is projected to increase the scale of mountain pine beetle outbreaks at high altitudes (Hicke et al. 2006), which potentially could have dramatic effects on the overall carbon balance within high-altitude whitebark pine communities (Kurz et al. 2008). Although the analysis presented within this paper provides a foundation for understanding the dynamics 
of white pine blister rust through the period 1968-2008, it also emphasizes a need for the continued study of the pathosystem dynamics within this sensitive high-altitude ecosystem into the future. Although it is likely that rapid biotic and abiotic changes will occur in whitebark pine communities, it is critical to continually reassess these dynamics in order to provide estimates of disease progression as well as to inform decisions about management solutions that buffer the negative effects of widespread mortality to whitebark pine trees, such as transplanting resistant individuals.

\section{CONCLUSION}

The formulation of our spatially implicit metapopulation model predicts the future levels of blister rust infection in whitebark pine at sites throughout the GYE by parameterizing a data set that spans 1968-2008. By conducting an analysis that compares four possible white pine blister rust dynamic infection models, we conclude that white pine blister rust operates through mechanisms that are strongly density dependent at the local level within individual sites, and more weakly density dependent on the background level of infection within the ecosystem as a whole. Results from our model are used to calculate the residence times for each of the infective stages of white pine blister rust, as well as the time to $90 \%$ infection throughout the ecosystem. Although we were not able to correlate environmental drivers with variation in blister rust infection at the site level, our analysis indicates that blister rust has pervaded most of the GYE, regardless of possible environmental barriers present within the 121 sites in our aggregated data set. The lack of correlation between heterogeneity in blister rust infection rates and environmental heterogeneity might indicate that the disease has pervaded all possible whitebark pine habitats in the GYE.

The modeling approach presented within this analysis could be applied to analyze infection dynamics in other systems where infected populations are relatively stationary (e.g., plants, amphibians) relative to the infection agent. Our approach is unique in that it accounts for infection dynamics at both the site and ecosystem levels that are driving the process of infection. Forest managers can utilize results from this study to both track the rate of infection within sites and analyze the average rates of white pine blister rust progression throughout the ecosystem. Results from this modeling analysis might be used to identify sites with slow rates of disease progression, which might indicate genetic resistance within certain populations. Additionally, these results might help to inform reforestation efforts by identifying the rates at which white pine blister rust infection is progressing. This study outlines a modeling approach that provides valuable information regarding site-level and ecosystem-wide infection dynamics that analyzed the past progression of blister rust and begins to identify its course into the future. The rapid progression of white pine blister rust spread in whitebark pine of the GYE should spark interest in ecological studies that begin to examine changes to the community-level dynamics that will likely ensue with the decline of whitebark pine.

Furthermore, the analysis and results presented within this study serve as an example of the benefits of datasharing agreements between different scientific groups. It is easy to imagine that the conclusions from this modeling analysis might not have been as robust had each of the data sets been modeled individually. Through collaboration, it was possible to increase both the temporal and spatial scope of our study to utilize the full breadth of information that was available regarding white pine blister rust in the GYE. As ecologists are increasingly faced with problems that bridge small scales with regional and global changes into the future, we emphasize that collaboration and data sharing can be an invaluable tool for ecological monitoring and modeling.

\section{ACKNOWLEDGMENTS}

The NASA Biodiversity and Ecological Forecasting program supported this research under award NNA07CN19A to R. L. Crabtree. Many thanks to Cathie Jean and the members of the Interagency Whitebark Pine Monitoring Working Group for providing access to data, insights for data interpretation, and advice for field study designs that were essential to this work. We also thank Gil Bohrer for helpful comments and suggestions in the modeling design, and members of the Yellowstone Ecological Research Center for assistance with data aggregation and fieldwork. Any use of trade, product, or firm names is for descriptive purposes only and does not imply endorsement by the U.S. Government.

\section{Literature Cited}

Arthur, J. C. 1934. Manual of the rusts in United States and Canada. Purdue Research Foundation, Lafayette, Indiana, USA.

Brown, J. K. M., and M. S. Hovmoller. 2002. Epidemiology: aerial dispersal of pathogens on the global and continental scales and its impact on plant disease. Science 297:537-541.

Campbell, E., and J. Antos. 2000. Distribution and severity of white pine blister rust and mountain pine beetle on whitebark pine in British Columbia. Canadian Journal of Forest Research 30:1051-1059.

Castello, J. D., D. J. Leopold, and P. J. Smallidge. 1995. Pathogens, patterns, and processes in forest ecosystems. BioScience 45:16-24.

Clark, J. S. 2007. Models for ecological data. Princeton University Press, Princeton, New Jersey, USA.

Ellison, A. M., et al. 2005. Loss of foundation species: consequences for the structure and dynamics of forested ecosystems. Frontiers in Ecology and the Environment $3: 479-486$

Frelich, L. E., and P. B. Reich. 1999. Neighborhood effects, disturbance severity, and community stability in forests. Ecosystems 2:151-166.

Gelfand, A. E., and S. K. Ghosh. 1998. Model choice: a minimum posterior predictive loss approach. Biometrika $85: 1-11$.

GYIWPMG (Greater Yellowstone Interagency Whitebark Pine Monitoring Group). 2007. Interagency whitebark pine monitoring protocol for the Greater Yellowstone Ecosystem. Greater Yellowstone Coordinating Committee, Bozeman, Montana, USA. 
Halligan, K. Q., R. L. Crabtree, and M. O. Jones. 2003. Hyperspectral data analysis of whitebark pine (Pinus albicaulis) and white pine blister rust (Cronartium ribicola) at four sites in the Greater Yellowstone Ecosystem. Yellowstone Ecological Research Center, Bozeman, Montana, USA.

Hatala, J. A., R. L. Crabtree, K. Q. Halligan, and P. R. Moorcroft. 2010. Landscape-scale patterns of forest pest and pathogen damage in the Greater Yellowstone Ecosystem. Remote Sensing of Environment 114:375-384.

Hicke, J. A., J. A. Logan, J. Powell, and D. S. Ojima. 2006. Changing temperatures influence suitability for modeled mountain pine beetle (Dendroctonus ponderosae) outbreaks in the western United States. Journal of Geophysical Research: Biogeosciences111:G02019. [doi:10.01029/ 02005JG000101]

Holdenrieder, O., M. Pautasso, P. J. Weisberg, and D. Lonsdale. 2004. Tree diseases and landscape processes: the challenge of landscape pathology. Trends in Ecology and Evolution 19:446-452.

Kendall, K. C. 1995. Whitebark pine sampling instructions for Ecodata and Survey Plots. U.S. Geologic Survey. USGS Rocky Mountain Science Center, Missoula, Montana, USA.

Kendall, K. C., and S. F. Arno. 1990. Whitebark pine: an important but endangered wildlife resource. Pages 264-273 in W. Schmidt and K. McDonald, editors. Proceedings of a Symposium on Whitebark Pine Ecosystems. U.S. Forest Service General Technical Report INT-270, USDA Forest Service Intermountain Research Center, Ogden, Utah, USA.

Kurz, W. A., C. C. Dymond, G. Stinson, G. J. Rampley, E. T. Neilson, A. L. Carroll, T. Ebata, and L. Safranyik. 2008. Mountain pine beetle and forest carbon feedback to climate change. Nature 452:987-990.

Logan, J. A., J. Regniere, and J. A. Powell. 2003. Assessing the impacts of global warming on forest pest dynamics. Frontiers in Ecology and Environment 1:130-137.

Maloy, O. C. 1997. White pine blister rust control in North America: a case history. Annual Review of Phytopathology 35:87-109.

McDonald, G. I., B. A. Richardson, P. J. Zambino, N. B. Klopfenstein, and M.-S. Kim. 2006. Pedicularis and Castille$j a$ are natural hosts of Cronartium ribicola in North America: a first report. Forest Pathology 36:73-82.

Moorcroft, P. R., S. W. Pacala, and M. A. Lewis. 2006. Potential role of natural enemies during tree range expansions following climate change. Journal of Theoretical Biology 241:601-616.

Newcomb, M. 2003. White pine blister rust, whitebark pine, and Ribes species in the Greater Yellowstone Area. Thesis. University of Montana, Missoula, Montana, USA.
O'Neill, R. V., R. H. Gardner, M. G. Turner, and W. H. Romme. 1992. Epidemiology theory and disturbance spread on landscapes. Landscape Ecology 7:19-26.

Park, A. W., S. Gubbins, and C. A. Gilligan. 2001. Invasion and persistence of plant parasites in a spatially structured host population. Oikos 94:162-174

Park, A. W., S. Gubbins, and C. A. Gilligan. 2002. Extinction times for closed epidemics: the effects of host spatial structure. Ecology Letters 5:747-755.

Six, D. L., and J. Adams. 2007. White pine blister rust severity and selection of individual whitebark pine by the mountain pine beetle (Coleoptera: Curculionidae, Scolytinae). Journal of Entomological Science 42:345-353.

Six, D. L., and M. Newcomb. 2005. A rapid system for rating white pine blister rust incidence, severity, and within-tree distribution in whitebark pine. Northwest Science 79:189195.

Smith, J. P., and J. T. Hoffman. 2000. Status of white pine blister rust in the Intermountain West. Western North American Naturalist 60:165-179.

Snell, W. H. 1942. The production of sporidia of Cronartium ribicola on cultivated red currants in relation to infection of white pine. American Journal of Botany 29:506-513.

Sniezko, R. A., D. F. Tomback, R. M. Rochefort, E. Goheen, R. Hunt, J. S. Beatty, M. Murray, and F. Betlejewski. 2004. Exotic pathogens, resistant seed and restoration of forest tree species in western North America. Pages 21-26 in K. L. Mergenthaler, J. E. Williams, and E. S. Jules, editors. Proceedings of the Second Conference on Klamath-Siskiyou Ecology. Field Institute, Cave Junction, Oregon, USA.

Spaulding, P. C. 1922. Investigations of the white pine blister rust. USDA Bulletin 957.

Tomback, D. F., S. F. Arno, and R. E. Keane. 2001. The compelling case for management intervention. Pages 3-25 in D. F. Tomback, S. F. Arno, and R. E. Keane, editors. Whitebark pine communities: ecology and restoration. Island Press, Washington, D.C., USA.

van Arsdel, E. P., A. J. Riker, and R. F. Patton. 1956. The effects of temperature and moisture on the spread of white pine blister rust. Phytopathology 46:307-318.

Yellowstone National Park. 1968-1971. Blister rust incidence survey, Reference FSH 5277 R1. U.S. National Park Service, Yellowstone Field Office, Mammoth, Wyoming, USA

Yi, C. K., and H. J. Kim. 1983. Studies on the host selectivity and pathogenicity of white pine blister fungus (Cronartium ribicola J.C. Fischer ex Rabenhorst) in Korea. Journal of the Korean Forest Society 62:76-81.

Zambino, P. J., B. A. Richardson, and G. I. McDonald. 2007. First report of the white pine blister rust fungus, Cronartium ribicola, on Pedicularis bracteosa. Plant Disease 91: 467.

APPENDIX

White pine blister rust model output for all 121 sites (Ecological Archives A021-050-A1).

\section{SUPPLEMENT}

Source code and model output for white pine blister rust Bayesian infection model (Ecological Archives A021-050-S1). 